\title{
Label-free intracellular transport measured by spatial light interference microscopy
}

\author{
Zhuo Wang, ${ }^{a}$ Larry Millet, ${ }^{b}$ Vincent Chan, ${ }^{c}$ Huafeng Ding, ${ }^{a}$ Martha U. Gillette,,${ }^{b, c}$ Rashid Bashir, ${ }^{a, c}$ and Gabriel Popescu ${ }^{a}$ \\ a University of Illinois at Urbana-Champaign, Department of Electrical and Computer Engineering, Beckman Institute \\ for Advanced Science \& Technology, Urbana, Illinois 61801 \\ buniversity of Illinois at Urbana-Champaign, Department of Cell and Developmental Biology, Urbana, Illinois 61801 \\ ${ }^{c}$ University of Illinois at Urbana-Champaign, Department of Bioengineering, Urbana, Illinois 61801
}

\begin{abstract}
We show that applying the Laplace operator to a speckle-free quantitative phase image reveals an unprecedented level of detail in cell structure, without the gradient artifacts associated with differential interference contrast microscopy, or photobleaching and phototoxicity limitations common in fluorescence microscopy. This method, referred to as Laplace phase microscopy, is an efficient tool for tracking vesicles and organelles in living cells. The principle is demonstrated by tracking organelles in cardiomyocytes and vesicles in neurites of hippocampal neurons, which to our knowledge are the first label-free diffusion measurements of the organelles in such cells. $\odot 2011$ Society of Photo-Optical Instrumentation Engineers (SPIE). [DOI: 10.1117/1.3549204]
\end{abstract}

Keywords: quantitative phase imaging; intrinsic particle tracking; interference microscopy; phase measurement.

Paper 10178SSR received Apr. 5, 2010; revised manuscript received Dec. 30, 2010; accepted for publication Jan. 3, 2011; published online Feb. 28, 2011.

\section{Introduction}

Particle tracking has drawn a lot of attention as a useful tool for rheology and micro-rheology studies. ${ }^{1,2}$ It is a popular way to evaluate the viscoelastic properties of micro-environments such as the cell cytoplasm, cell membrane, polymer solutions, etc. For cellular studies the probing beads are inserted into the cells or attached to the membrane, under the general assumption that they do not alter the normal physiology of the cell. Alternatively, fluorescence markers can be used for particle tracking. However, the fluorescence signal is usually weak, suffers from photobleaching, and may produce phototoxicity under long term observation. Therefore, it is valuable to develop label-free methods for tracking intrinsic particles within cells. Since the cells are usually transparent, or termed as phase object, a method based on phase information is required for imaging.

Quantitative phase imaging (QPI) of cells and tissues has recently become a dynamic area of study (for a recent review, see Ref. 3). Compared to other qualitative phase imaging methods such as phase contrast and differential interference contrast, QPI has the ability to quantify the optical path-length map of a light field passing through the specimen. Due to its ability to render label-free, quantitative, and nanoscale information from live cells, a rapidly increasing number of QPI methods have been developed within the past decade or so. ${ }^{4-17}$ Despite all these developments, the range of QPI applications in biology has been largely limited to red blood cell imaging ${ }^{18-21}$ or assessment of global cell parameters such as dry mass, ${ }^{12,22-25}$ average refractive index,,$^{26,27}$ and statistical parameters of tissue slices. ${ }^{14}$ In our opinion, this current limitation is due to two main reasons. First, because of the speckle generated by the high coherence of the illuminating light (typically lasers), the contrast to noise

Address all correspondence to: Gabriel Popescu, University of Illinois at Urbana-Champaign, Department of Electrical and Computer Engineering, Beckman Institute for Advanced Science \& Technology, Urbana, Illinois 61801. Tel: 01-217-333-4840; Fax: 01-217-244-1995; E-mail: gpopescu@illinois.edu ratio in QPI images has never matched that exhibited in white light techniques such as phase contrast microscopy. Second, like all interferometric methods, the experimental arrangements tend to be rather complex, of high maintenance, which limits their indepth biological applicability. A number of QPI-based tracking methods were reported recently. ${ }^{28-30}$ However, in these previous reports, large beads or the cells themselves were tracked.

On the other hand, qualitative methods such as phase contrast or differential interference contrast (DIC) provide better contrast to noise ratio, but their application to intrinsic particle tracking is limited as well.

Toward this end, we have recently developed spatial light interference microscopy (SLIM) as a novel, highly sensitive QPI method, which is advantageous for enabling quantitative assessment of specimen structure and cell dynamics. ${ }^{31}$ SLIM combines Zernike's white light phase contrast microscopy which reveals the intrinsic contrast of transparent samples ${ }^{32}$ with Gabor's holography, ${ }^{33}$ by rendering quantitative phase maps across the specimen.

In this paper, we exploit the exquisite spatial sensitivity of SLIM and demonstrate that the Laplace operator can be used to reveal a high-detail quantitative phase image without gradient artifacts common to DIC microscopy. In particular, this imaging approach, termed Laplace phase microscopy (LPM), allows us to quantify intracellular transport without the typical need for exogenous contrast agents. This opens up new avenues for particle tracking, which has been largely limited to fluorescently labeled tracers. ${ }^{1}$ We show that LPM can study transport in live cells over very broad time scales, unlimited by photobleaching or photoxicity, as demonstrated by tracking organelles in hippocampal neurons and cardiomyocytes. Our measurements indicate a diffusive regime for the particle motion, from which the diffusion coefficient can be extracted quantitatively.

$1083-3668 / 2011 / 16(2) / 026019 / 9 / \$ 25.00$ (C) 2011 SPIE 


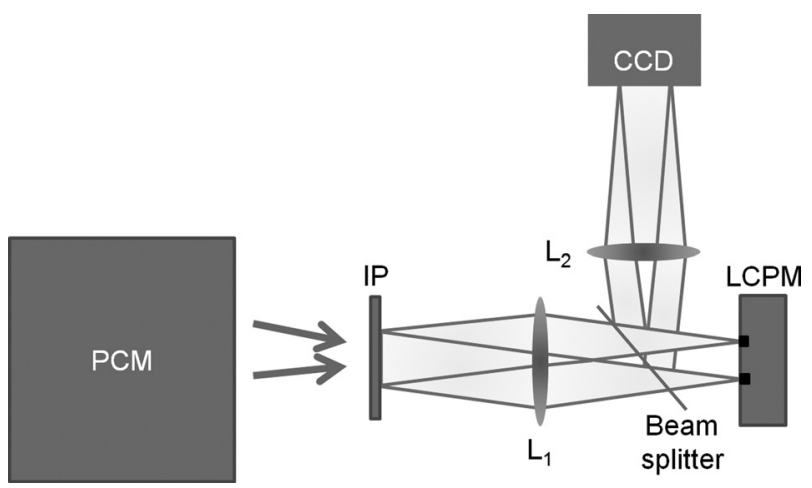

Fig. 1 Schematic setup for SLIM. The black dots on the LCPM show the cross section through the ring along which the unscattered light is distributed.

\section{Experimental Setup}

The experimental setup is described in detail elsewhere. ${ }^{34}$ Here we briefly review its principle of operation (Fig. 1). The SLIM module is attached to a commercial phase contrast microscope (Axio Observer Z1, Zeiss, in this case). The image outputted by the microscope is Fourier transformed by lens $L_{1}$, which essentially images the objective phase contrast ring onto the surface of a liquid crystal phase modulator (LCPM). This device can modulate the phase difference $\Delta \varphi$ between the scattered and unscattered light in increments of $\pi / 2$, i.e., $\Delta \varphi=0, \pi / 2, \pi$, and $3 \pi / 2$. The Fourier lens $L_{2}$ completes a (folded) $4 f$ system, such that the conjugate image plane is reconstructed at the CCD plane. By recording one intensity image for each of the four phase shifts introduced by LCPM, we render the final, quantitative phase image associated with the sample.

SLIM provides several advantages over existing techniques, including speckle-free images, which allows for spatially sensitive optical path-length measurement $(0.3 \mathrm{~nm})$, common path geometry that enables temporally sensitive optical path-length measurement $(0.03 \mathrm{~nm})$, and 3D tomographic rendering of transparent structures. ${ }^{31}$ Further, due to the broadband illumination spectrum, SLIM grants immediate potential for spectroscopic (i.e., phase dispersion) imaging. SLIM is likely to make a broad impact by implementation with existing phase contrast microscopes and overlaying with fluorescence imaging for multimodal, in-depth biological studies.

\section{Data Analysis}

Figure 2(a) (Video 1) shows an example of a quantitative phase image, $\phi(x, y)$, of cardiomyocytes, which were obtained from 2-day old neonatal Sprague-Dawley rats (Harlan Laboratories, Inc.) using an approved protocol by the Institutional Animal
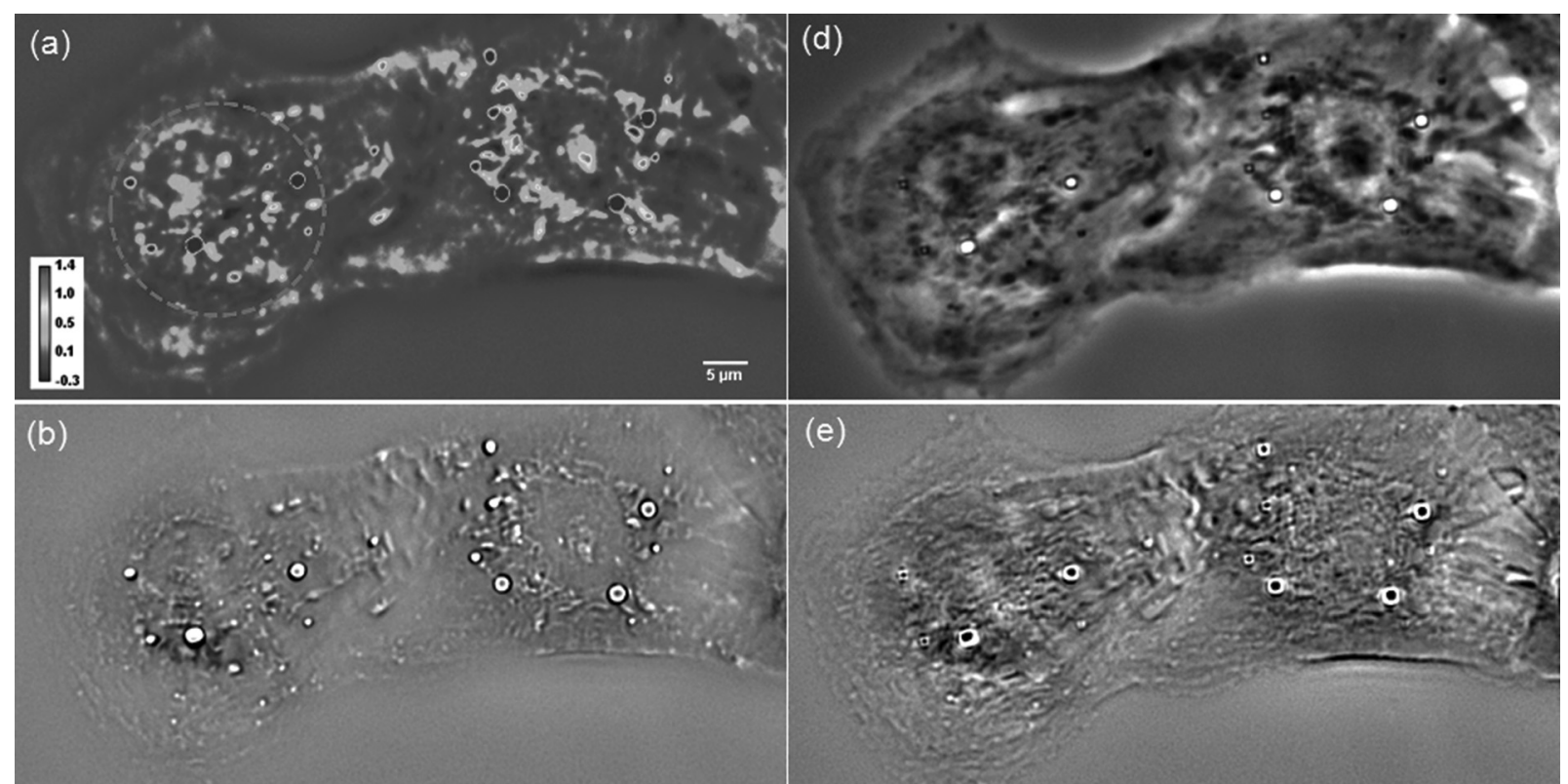

(e)

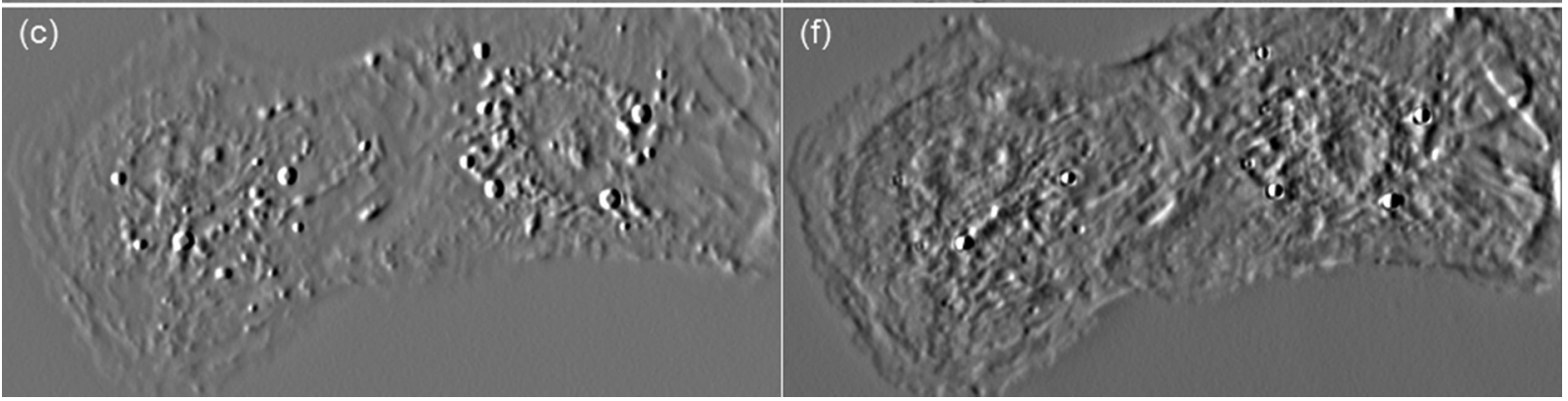

Fig. 2 (a) SLIM image of two cardiac myocytes in culture; color bar indicates phase shift in radians (Video 1). The dashed circles show the cell nuclei. The objective is ZEISS Plan-Neofluar 40X/0.75. (b) Laplacian of (a) Video 1. (c) Simulated DIC, as gradient in $X$ direction of (a). (d) Phase contrast image. (e) Laplacian of $(d)$. (f) Gradient in $X$ direction of (d). 

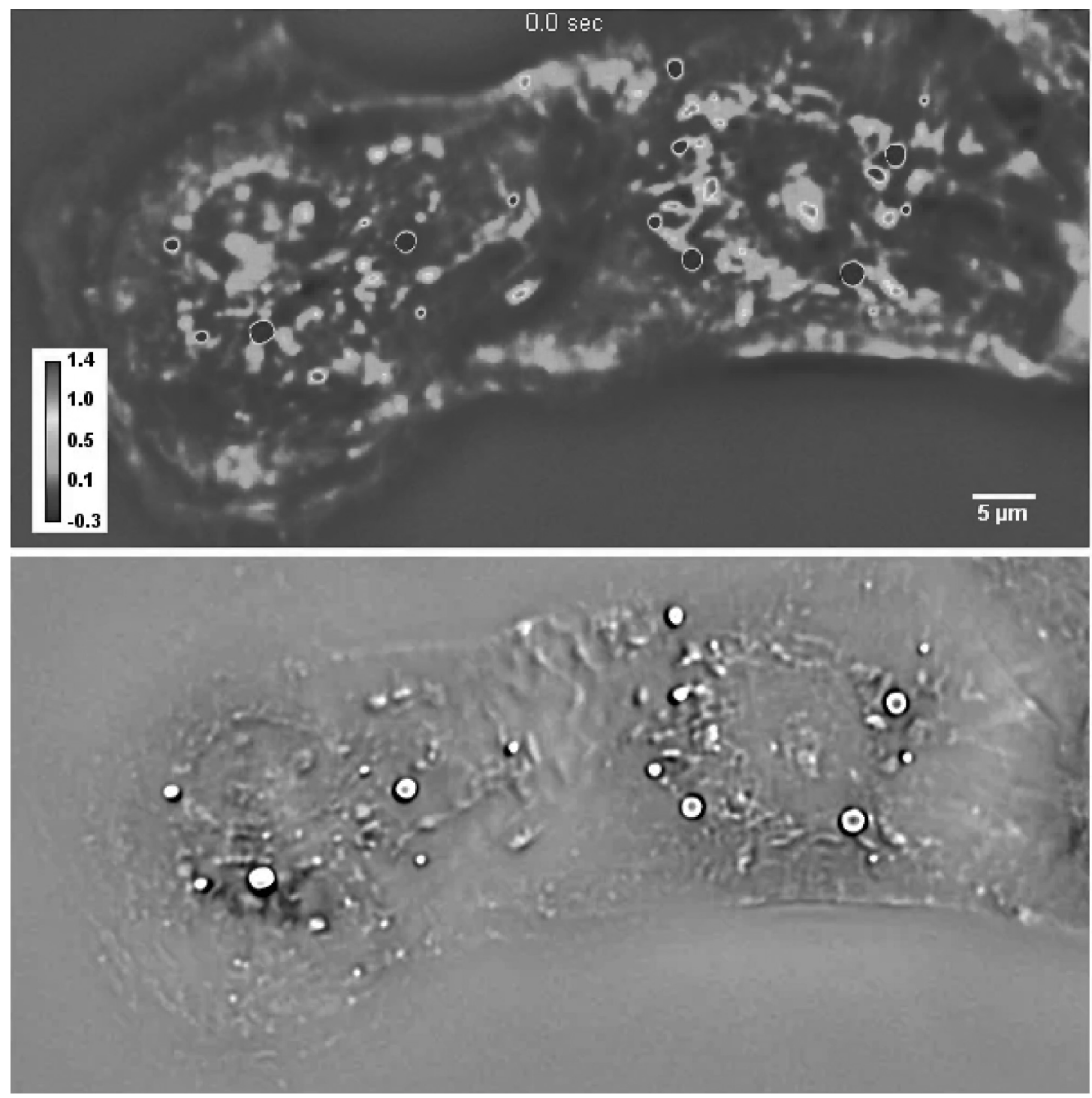

Video 1 SLIM and LPM imaging of cardiomyocytes in culture. Cells are beating simultaneously. Particles are revealed and traced by LPM (QuickTime, 7.6 MB). [URL: http://dx.doi.org/10.1117/1.3549204.1]

Care and Use Committee. Briefly, whole hearts were excised ${ }^{35}$ in ice-cold Hanks balanced salt solution (HBSS) buffer, the atria were removed, and the remaining ventricles were quartered and digested in $0.05 \%(\mathrm{w} / \mathrm{v})$ trypsin (Worthington Biochemicals) with gentle rotation $\left(4^{\circ} \mathrm{C}, 16 \mathrm{~h}\right)$. To inhibit trypsin digestion, growth media (DMEM with $10 \%$ fetal bovine serum) was added for $5 \mathrm{~min}$ at $37^{\circ} \mathrm{C}$. After discarding the supernatant, $0.1 \%$ (w/v) purified type II collagenase (Worthington Biochemicals) was added for $45 \mathrm{~min}$ while rotating at $37^{\circ} \mathrm{C}$. The cardiac tissue was gently triturated to mechanically loosen the cells, filtered through a cell strainer, and centrifuged at $150 \times \mathrm{g}$ for $10 \mathrm{~min}$. The cell pellet was resuspended in $37^{\circ} \mathrm{C}$ growth media and pre-plated for $1 \mathrm{~h}$ to enrich the suspension for cardiac myocytes.

We computed the LPM image [Fig. 2(b) and Video 1] via the Laplace operator

$$
\nabla^{2} \phi(x, y)=\frac{\partial^{2} \phi}{\partial x^{2}}+\frac{\partial^{2} \phi}{\partial y^{2}} .
$$

In order to filter out the high-frequency noise, which is amplified by the Laplace operator, we first convolved the images with a Gaussian kernel that is narrower than the diffraction spot $(1.22 \lambda / \mathrm{NA}=0.86 \mu \mathrm{m})$. Since the Laplacian is a linear operator, this is equivalent to a convolution of the image with the Laplacian of a Gaussian. ${ }^{36}$ Here, the input image is represented in discrete pixels, a discrete kernel that can approximate second order derivatives needs to be used. One possible convolution kernel is a $3 \times 3$ matrix: ${ }^{36}$

$$
\left[\begin{array}{rrr}
0 & -1 & 0 \\
-1 & 4 & -1 \\
0 & -1 & 0
\end{array}\right]
$$

As evident from this image, the Laplace operator is able to clearly define the organelle structures within the cell. It is known that heart cells are very active, i.e., energy-consuming; therefore this type of cell is rich in mitochondria, which are responsible for the energy supply of the cell metabolism. ${ }^{37}$ Mitochon- 
Wang et al.: Label-free intracellular transport measured by spatial light interference microscopy
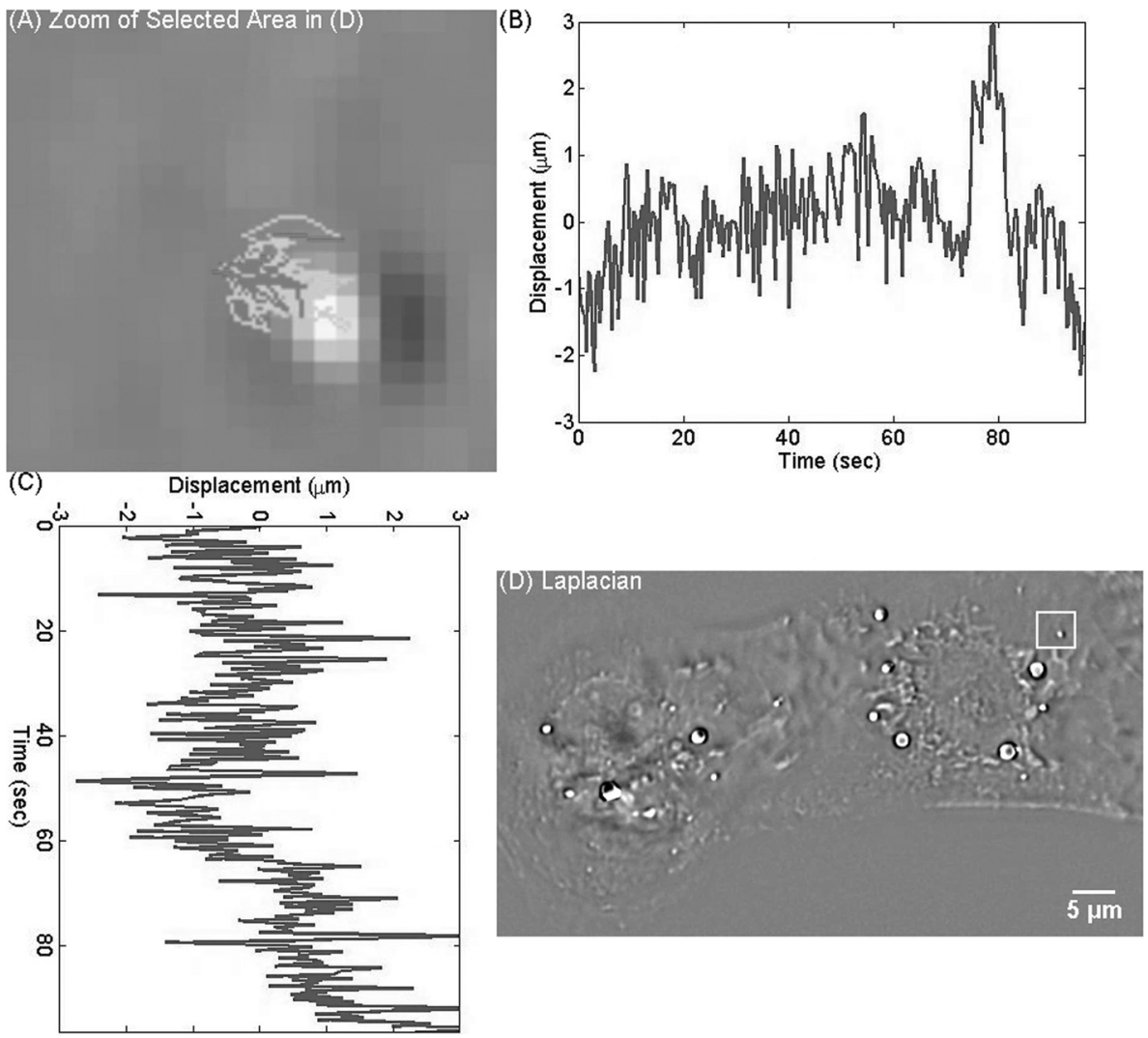

Video 2 Particle tracking of organelles in cardiomyocytes using LPM (QuickTime, 7.7 MB). [URL: http://dx.doi.org/10.1117/1.3549204.2]
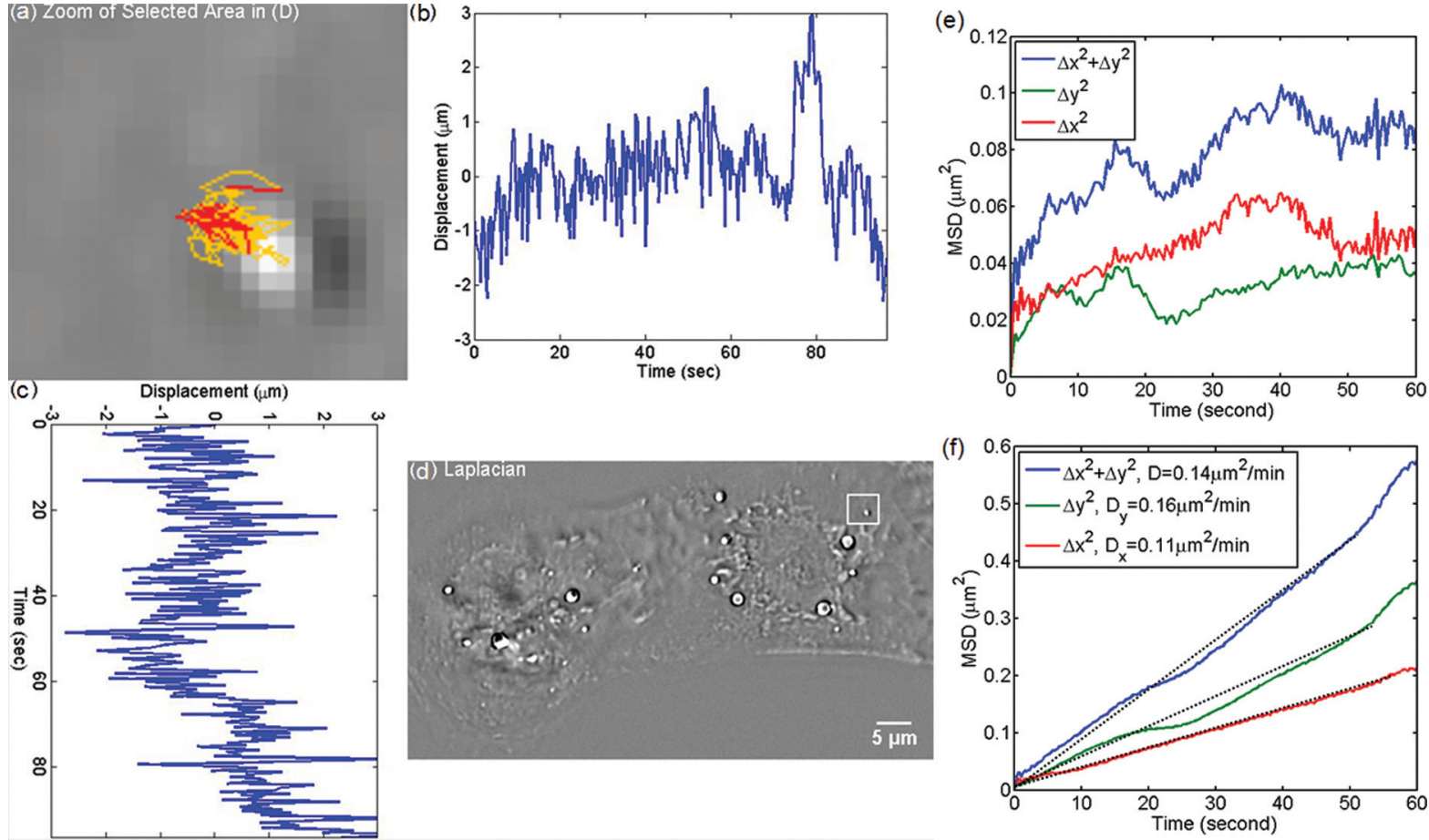

Fig. 3 MSD measurement for particles within the cardiac myocytes in Fig. 2. (a) Zoom into the selected area shown in (d); (b) Displacement in $Y$ direction; (c) Displacement in $X$ direction. (d) Laplacian of the phase map [same as Fig. 2(b)]; (e) MSD for the particle shown in (a). (f) MSD ensemble-averaged over 15 particles in (d). The respective movie can be found in the supplemental material (Video 2). 


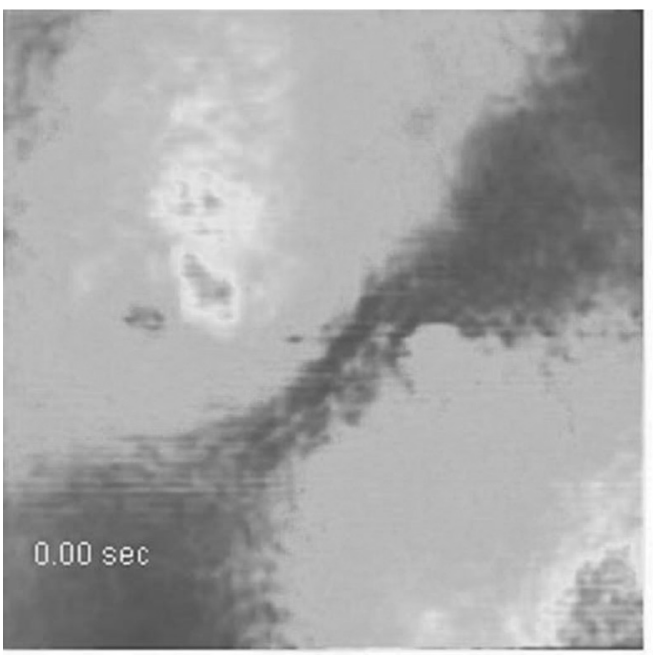

(a)

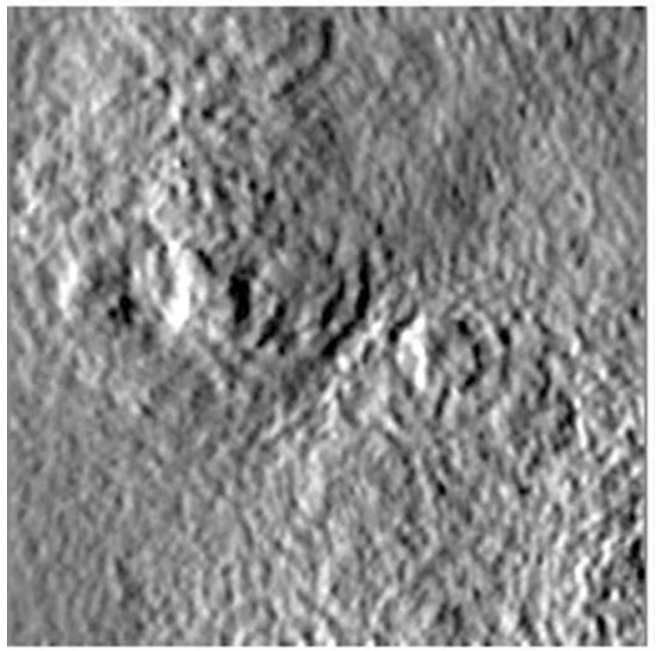

(c)

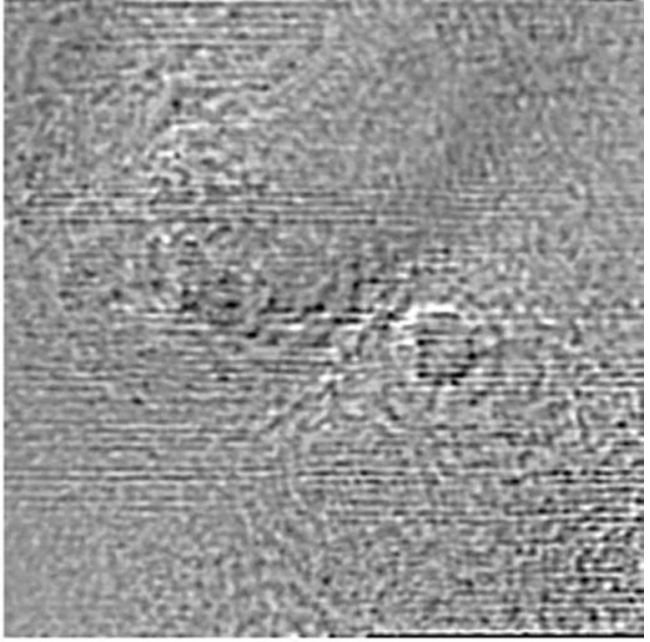

(b)

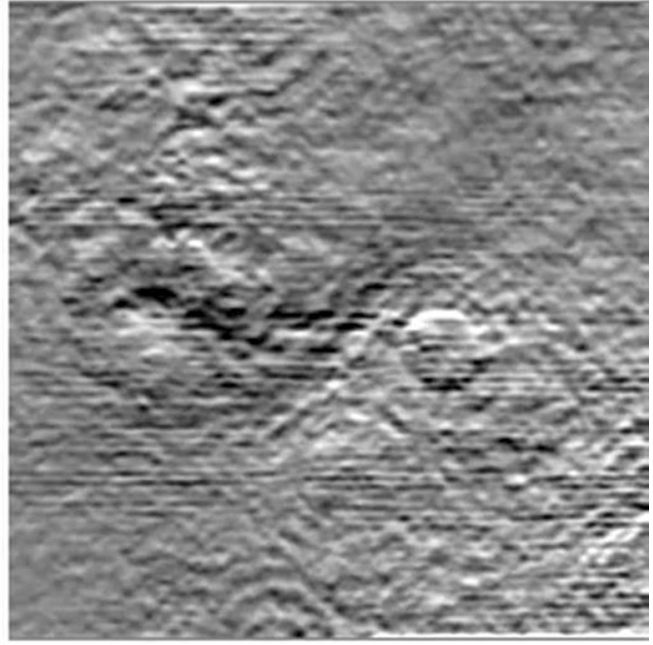

(d)

Video 3 DPM imaging of the cardiomyocytes in culture. No particles can be resolved. (a) Phase map, (b) Laplacian of phase map, (c) gradient in vertical direction, (d) gradient in horizontal direction. The field of view is $32 \mu \mathrm{m} \times 32 \mu \mathrm{m}$ (QuickTime, 5.9 MB). [URL: http://dx.doi.org/10.1117/1.3549204.3]

dria are most likely the predominant type of visible organelles, especially in the area surrounding the cell nucleus [indicated by the circular region in Fig. 2(a)]. For comparison, we also computed a "synthetic" differential interference contrast (DIC or Nomarski) image [Fig. 2(c)], via the gradient of the quantitative phase image,

$$
\bar{\nabla} \phi(x, y)=\frac{\partial \phi}{\partial x} \widehat{\mathbf{x}}+\frac{\partial \phi}{\partial x} \widehat{\mathbf{y}},
$$

where $\mathbf{x}$ and $\mathbf{y}$ are the unit vectors along the two coordinates. While DIC can reveal intracellular particles, artifacts ("shadow" effects) are typically introduced due to the rapid change in sign of the first order derivatives, as visible in Fig. 2(c). By contrast, the LPM image is free of such artifacts, because it is based on a second order derivative [Eq. (1)]. Figure 2(d) shows the phase contrast image of the same cell, which reveals the difficulties associated with particle tracking due to the reduced contrast for small particles. Because phase contrast mixes the intensity and phase information suffers from phase ambiguity and is qualitative, the Laplacian of a phase contrast image is of much lower quality compared to LPM, as shown in Fig. 2(e). Thus, LPM offers valuable opportunities for tracking these particles inside live cells, which in turn reports on the statistics of the organelle transport and on their diffusion coefficients. The measured irradiance at the sample plane is about $1 \mathrm{nW} / \mu \mathrm{m}^{2}$. The exposure time is usually 10 to $50 \mathrm{~ms}$. Thus, this irradiance is 6 to 7 orders of magnitude below that of typical confocal microscopy. ${ }^{38}$ Unlike imaging fluorescently tagged organelles, in LPM the imaging can be performed over arbitrarily long time scales without limitations due to photobleaching or phototoxicity.

Figure 3 demonstrates the procedure of quantifying intracellular diffusion via LPM. LPM images of a pair of beating cardiomyocytes in culture were recorded for more than a minute at a rate of 2.6 frames/s. The speed is currently limited by our acquisition camera which has a frame limit of 11 frames/s at full resolution. The organelle diffusion coefficient $D$ is extracted from the measured mean squared displacement (MSD) of the particles, and is defined as ${ }^{2}$

$$
\Delta r^{2}(\tau)=\left\langle[x(t+\tau)-x(t)]^{2}+[y(t+\tau)-y(t)]^{2}\right\rangle .
$$

In Eq. (3), the angular bracket stands for temporal and ensemble average. For a diffusion process, the mean squared displacement grows linearly in time, $\Delta r^{2}(\tau)=2 n D \tau$, where $n$ indicates the 
dimensionality, i.e., $n=1,2$, and 3 for diffusion in 1,2 , and 3 dimensions, respectively. ${ }^{2}$ Thus, the slope of $\Delta r^{2}$ reveals the diffusion coefficient.

Figures 3(a)-3(d) illustrate the tracking of a single particle within the cell. Figure 3(a) shows the magnified particle in the selected area of Fig. 3(d). Particles are traced using an automatic algorithm implemented in Java. The tracking algorithm may lose the particle at certain frames (e.g., due to the particle going out of focus) and find the particle again in later frames. If the algorithm identifies the same particles and relinks the traces, the link (an indication of skipped frames) is in red and the trace is in yellow [e.g., red in Fig. 3(a) and Video 3]. This will not cause any problem in the calculation of MSD because it is a time and ensemble average and is not sensitive to the individual frame loss. Figures 3(b) and 3(c) show the displacement of the particle in the $Y$ and $X$ directions, respectively. The MSD for the specific particle shown in Fig. 3(e) shows no specific trend at all, as expected. It can be seen that the high frequency component of the signal is due to the cell beating. However, if we analyze all 15 particles in Fig. 3(d) and perform an ensemble average, the MSD is linear which clearly shows a diffusive movement, as summarized in Fig. 3(f). The results demonstrate that the high-frequency beating signal is averaged out and that a linear dependence is obtained. The resulting diffusion along $y$ is slightly smaller than along $x$, which might be explained by the beating signal propagation between the two cells, almost parallel to the $x$-axis. Note that the overall value, $D=0.14 \mu \mathrm{m}^{2} / \mathrm{min}$, obtained for the diffusion coefficient, $D=\left(D_{x}+D_{y}\right) / 2$, is approximately 188 times smaller than that predicted by the Stokes-Einstein equation for a $1-\mu \mathrm{m}$ particle diffusing in water at room temperature. ${ }^{39}$ This reduced diffusion of organelles within the cells can be understood by realizing that organelles occupy a crowded space, populated by cytoskeletal network and macromolecules, which makes the effective viscosity significantly higher than that of water. ${ }^{40}$ For comparison, we also captured the cardiomyocytes in culture with diffraction phase microscopy (DPM) ${ }^{41}$ an established laser technique for quantitative phase imaging (Video 3). As evident in the movie, although the acquisition rate is faster, no particles can be resolved at all. It is clear that by simply applying a Laplacian operator to a laser-based quantitative phase image is not useful for tracking intrinsic particles.

Further, we applied the LPM method to vesicles in hippocampal neurites. Recently, tracking of synaptic vesicles with far-field optical nanoscopy has been demonstrated. ${ }^{42}$ Primary neuronal cultures from the postnatal rat were generated on glass bottomed dishes for imaging analysis based on the previously defined protocol. Briefly, postnatal hippocampal neurons were isolated from postnatal rats through enzymatic digestion, tissue rinse, dissociation, and centrifugation, followed by resuspension and plating in supplemented neurobasal media until imaging. Prior to imaging, neuron media was replaced with supplemented, $\mathrm{CO}_{2}$ independent media Hibernate-A. ${ }^{43}$

With the high sensitivity of SLIM, the vesicles in neurites can be resolved, as seen from Fig. 4 (Video 4). In our samples, SLIM images were acquired at 4 frames/min. The phase map shown [Fig. 4] for the dashed line includes five temporal traces [Figs. 4(a) and 4(b) ], trace 1 is the background, 2 and 4 are two large processes, and 3 and 5 are two small processes. The optical path length changes for individual traces are shown in Fig. 4(b),
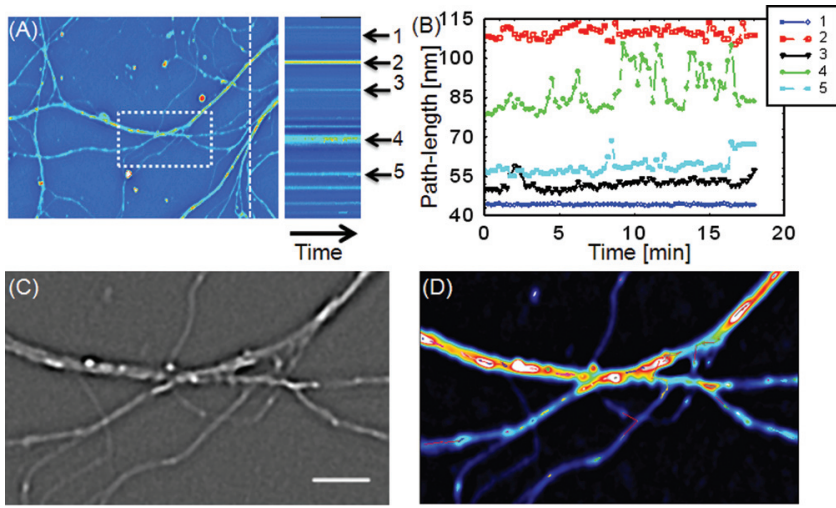

(E)

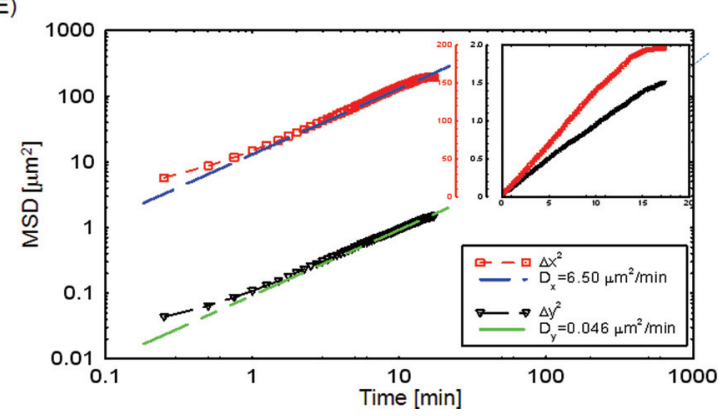

Fig. 4 Particle transport in neurites of a hippocampal neuron processor network. The objective is ZEISS Plan-Neofluar 40X/0.75. (a) Phase map of the neuron network. The arrows 1 to 5 show the time-traces of the corresponding points along the dashed line. The whole field of view is $100 \mu \mathrm{m} \times 75 \mu \mathrm{m}$. The objective used is Zeiss Plan-Neofluar $40 \times / 0.75$. (b) Optical path length change in time for the five points indicated in (a). Peaks in the point traces correspond to phase shifts associated with (fast) organelle traffic. (c) Laplacian of the selected area in (a). The scale bar is $5 \mu \mathrm{m}$. (d) Phase map of the same area as in (c), with some particle traces shown in fine lines. (e) Log-log plot of the MSD for 70 individual particles in (d). Since the particles are confined in the $Y$ direction, the diffusion coefficient for this direction is 2 orders of magnitude smaller than for the other direction. The inset shows the same MSD curves in linear representation and two $Y$ axes. See Video 4 for details.

where the background stays very stable, and the peaks in the figure correspond to the particles in transport. Since the optical path length change corresponds to the dry mass, the heights of the bumps are indicators of the protein mass under transport. Evident from Fig. 4(b) (Video 4), SLIM is sensitive enough to display the optical 10 to $20 \mathrm{~nm}$ path-length changes associated with particles in transport. Figure 4(c) is the Laplacian of the selected area in Fig. 4(a). The particles with the traces are shown in Fig. 4(d).

Neuronal processes demonstrate bidirectional transport of cargo (i.e., synaptic vesicle precursors, mitochondria, piccolobassoon transport vesicles, signaling endosomal organelles, and translation machinery) to and from the soma and distal tips. ${ }^{44-46}$ As seen in the movie, a subset of particles exhibit a fast, directed (nondiffusive) motion. However, for our mean squared displacement analysis, we retain only particle trajectories that survive throughout the entire measurement window of $18 \mathrm{~min}$. These long-time trajectories exhibit again diffusive motion, as indicated by the very strong linear dependence shown in the inset of Fig. 4(e). To our knowledge, these are the first label-free diffusion measurements in neurons. As expected, the displacements parallel to the $y$-axis are approximately 2 orders 

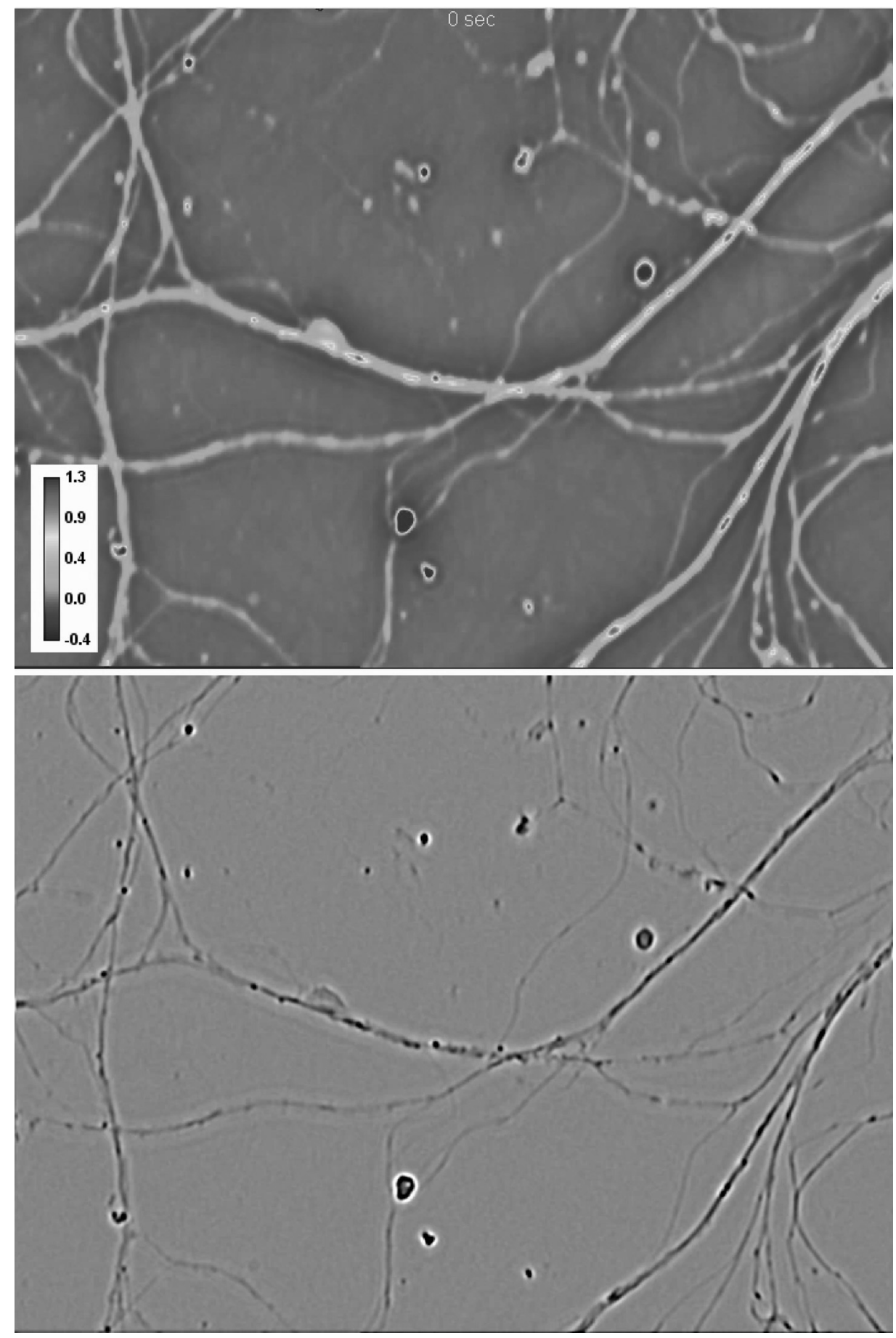

Video 4 SLIM and LPM imaging of live hippocampal neuron. Neurons and putative glia in primary cell culture. Vesicle transports in hippocampal neurites are emphasized and traced using LPM. The contrast of LPM is inverted for better viewing purpose (QuickTime, $3.0 \mathrm{MB}$ ). [URL: http://dx.doi.org/10.1117/1.3549204.4]

of magnitude smaller than the $x$-axis displacements because the neurites are largely parallel to the $x$-axis. Because neurons have numerous long neurites, the surface area of a neuron far exceeds that of other cells by as much as 10 to 1000 times. ${ }^{47}$ Thus, the structure and function of the neuron has high demands on cellular resources, and the active transport of cellular resources is needed to maintain these demands. Most axonal transport velocities range between 0.5 to $5.0 \mu \mathrm{m} / \mathrm{s} .{ }^{45}$ From our data, the directional transport velocity is about $0.5 \mu \mathrm{m} / \mathrm{s}$ and the diffusion coefficients $D_{x}=6.50 \mu \mathrm{m}^{2} / \mathrm{min}$ and $D_{y}=0.046 \mu \mathrm{m}^{2} / \mathrm{min}$. Thus, the diffusion process in dendrites is largely one-dimensional, as obvious from the movie. Note that the diffusion coefficient in neurons is significantly larger than that in the heart cells, $D=0.14 \mu \mathrm{m}^{2} / \mathrm{min}$, which indicates that the transport in neurons is more active. This result is consistent with the neuronal function that involves mass transport over large distances. 


\section{Conclusion}

In summary, we demonstrated LPM as an efficient method for revealing and tracking organelles within unstained cells such as cardiomyocytes and neurons. To our knowledge, these are the first label-free diffusion measurements in such cells. Based on the advance of spatial light interference microscopy, ${ }^{31}$ this approach may help unravel important open questions regarding intracellular transport, which modulates cell development and cell signaling. It will also facilitate the study of the viscoelastic properties of the cells. In principle, the Laplacian operator can be applied to any quantitative phase image. However, we note that this operation enhances the high-frequency noise, e.g., noise due to speckles, which may reduce the overall utility of this operation (see Video 3). Laplace phase microscopy, Fourier phase microscopy, ${ }^{9}$ Hilbert phase microscopy, ${ }^{15}$ and Fresnel particle tracking ${ }^{28}$ are examples of optical techniques that exploit the ability of mathematical operators (from which they borrow their names) to enhance optical imaging.

\section{Acknowledgments}

This research was supported in part by NSF CAREER 08-46660 (to G.P.) and Grainger Foundation (to G.P.). M.U.G, R.B., and G.P. are supported by the NSF-STC CBET-0939511. M.U.G. is supported by NIH grants HL 086870 and MH 085220. L.M. was supported by NIH/HD007333 Developmental Psychobiology and Neurobiology Training Grant. V.C. was supported by the NSF-STC CBET-0939511 and by a cooperative agreement that was awarded and administered by the US Army Medical Research \& Materiel Command (USAMRMC) and the Telemedicine \& Advanced Technology Research Center (TATRC), under Contract No. W81XWH0810701 (to R.B.).

\section{References}

1. D. Wirtz, "Particle-Tracking Microrheology of Living Cells: Principles and Applications," Ann. Rev. Biophys. 38, 301-326 (2009).

2. T. A. Waigh, "Microrheology of complex fluids," Rep. Prog. Phys. $\mathbf{6 8}$, 685-742 (2005).

3. G. Popescu, "Quantitative phase imaging of nanoscale cell structure and dynamics," in Methods in Cell Biology, P. J. Bhanu, Ed., p. 87, Elsevier, Burlington, MA (2008).

4. M. A. Choma, A. K. Ellerbee, C. H. Yang, T. L. Creazzo, and J. A. Izatt, "Spectral-domain phase microscopy," Opt. Lett. 30, 1162-1164 (2005).

5. C. Joo, T. Akkin, B. Cense, B. H. Park, and J. E. de Boer, "Spectraldomain optical coherence phase microscopy for quantitative phasecontrast imaging," Opt. Lett. 30, 2131-2133 (2005).

6. D. Paganin and K. A. Nugent, "Noninterferometric phase imaging with partially coherent light," Phys. Rev. Lett. 80, 2586-2589 (1998).

7. A. Barty, K. A. Nugent, D. Paganin, and A. Roberts, "Quantitative optical phase microscopy," Opt. Lett. 23, 817-819 (1998)

8. E. D. Barone-Nugent, A. Barty, and K. A. Nugent, "Quantitative phase-amplitude microscopy I: optical microscopy," J. Microscopy 206, 194-203 (2002).

9. G. Popescu, L. P. Deflores, J. C. Vaughan, K. Badizadegan, H. Iwai, R. R. Dasari, and M. S. Feld, "Fourier phase microscopy for investigation of biological structures and dynamics,"Opt. Lett. 29, 2503-2505 (2004).

10. W. S. Rockward, A. L. Thomas, B. Zhao, and C. A. DiMarzio, "Quantitative phase measurements using optical quadrature microscopy,"Appl. Opt. 47, 1684-1696 (2008).

11. Z. Wang, L. J. Millet, M. U. Gillette, and G. Popescu, "Jones phase microscopy of transparent and anisotropic samples," Opt. Lett. 33, 1270-1272 (2008).
12. D. Zicha and G. A. Dunn, "An Image-Processing System For Cell Behavior Studies In Subconfluent Cultures," J. Microscopy 179, 11-21 (1995).

13. P. Marquet, B. Rappaz, P. J. Magistretti, E. Cuche, Y. Emery, T. Colomb, and C. Depeursinge, "Digital holographic microscopy: a noninvasive contrast imaging technique allowing quantitative visualization of living cells with subwavelength axial accuracy," Opt. Lett. 30, 468-470 (2005)

14. H. Ding, Z. Wang, F. Nguyen, S. A. Boppart, and G. Popescu, "Fourier transform light scattering of inhomogeneous and dynamic structures,"Phys. Rev. Lett. 101, 238102 (2008).

15. T. Ikeda, G. Popescu, R. R. Dasari, and M. S. Feld, "Hilbert phase microscopy for investigating fast dynamics in transparent systems," Opt. Lett. 30, 1165-1168 (2005).

16. J. G. Wu, Z. Yaqoob, X. Heng, L. M. Lee, X. Q. Cui, and C. H. Yang, "Full field phase imaging using a harmonically matched diffraction grating pair based homodyne quadrature interferometer," Appl. Phys. Lett. 90, 151123 (2007).

17. N. T. Shaked, Y. Z. Zhu, N. Badie, N. Bursac, and A. Wax, "Reflective interferometric chamber for quantitative phase imaging of biological sample dynamics," J. Biomed, Opt. 15, 030503 (2010).

18. G. Popescu, T. Ikeda, K. Goda, C. A. Best-Popescu, M. Laposata, S. Manley, R. R. Dasari, K. Badizadegan, and M. S. Feld, "Optical measurement of cell membrane tension,"Phys. Rev. Lett. 97, 218101 (2006).

19. Y. K. Park, C. A. Best, K. Badizadegan, R. R. Dasari, M. S. Feld, T. Kuriabova, M. L. Henle, A. J. Levine, and G. Popescu, "Measurement of red blood cell mechanics during morphological changes," Proc. Natl. Acad. Sci. U.S.A. 107, 6731-6736 (2010).

20. Y. K. Park, C. A. Best, T. Auth, N. Gov, S. A. Safran, G. Popescu, S. Suresh, and M. S. Feld, "Metabolic remodeling of the human red blood cell membran,"Proc. Natl. Acad. Sci. U.S.A. 107, 1289 (2010).

21. Y. K. Park, M. Diez-Silva, G. Popescu, G. Lykotrafitis, W. Choi, M. S. Feld, and S. Suresh, "Refractive index maps and membrane dynamics of human red blood cells parasitized by Plasmodium falciparum," Proc. Natl. Acad. Sci. U.S.A. 105, 13730-13735 (2008).

22. G. Popescu, Y. Park, N. Lue, C. Best-Popescu, L. Deflores, R. R. Dasari, M. S. Feld, and K. Badizadegan, "Optical imaging of cell mass and growth dynamics," Am. J. Physiol. Cell. Physiol. 295, C538-C544 (2008).

23. H. G. Davies and M. H. Wilkins, "Interference microscopy and mass determination," Nature (London) 169, 541-541 (1952).

24. R. Barer, "Interference microscopy and mass determination," Nature (London) 169, 366-367 (1952).

25. B. Rappaz, E. Cano, T. Colomb, J. Kuhn, C. Depeursinge, V. Simanis, P. J. Magistretti, and P. Marquet, "Noninvasive characterization of the fission yeast cell cycle by monitoring dry mass with digital holographic microscopy,"J. Biomed. Opt. 14, 034049 (2009).

26. N. Lue, G. Popescu, T. Ikeda, R. R. Dasari, K. Badizadegan, and M. S. Feld, "Live cell refractometry using microfluidic devices," Opt. Lett. 31, 2759-2761 (2006)

27. C. L. Curl, C. J. Bellair, T. Harris, B. E. Allman, P. J. Harris, A. G. Stewart, A. Roberts, K. A. Nugent, and L. M. D. Delbridge, "Refractive index measurement in viable cells using quantitative phase-amplitude microscopy and confocal microscopy," Cytometry, Part A 65A, 88-92 (2005).

28. Y. K. Park, G. Popescu, R. R. Dasari, K. Badizadegan, and M. S. Feld, "Fresnel particle tracking in three dimensions using diffraction phase microscopy,"Opt. Lett. 32, 811-813 (2007).

29. P. Langehanenberg, L. Ivanova, I. Bernhardt, S. Ketelhut, A. Vollmer, D. Dirksen, G. Georgiev, G. von Bally, and B. Kemper, "Automated three-dimensional tracking of living cells by digital holographic microscopy," Biomed. Opt. 14, 014018 (2009).

30. J. Sheng, E. Malkiel, J. Katz, J. Adolf, R. Belas, and A. R. Place, "Digital holographic microscopy reveals prey-induced changes in swimming behavior of predatory dinoflagellates," Proc. Natl. Acad. Sci. U.S.A. 104, 17512-17517 (2007).

31. Z. Wang, K. Tangella, and G. Popescu, "Tissue refractive index as marker for disease," Nat. Photonics (submitted).

32. F. Zernike, "How I discovered phase contrast," Science 121, 345-349 (1955). 
33. D. Gabor, “A new microscopic principle," Nature (London) 161, 777778 (1948).

34. Z. Wang, L. Millet, M. Mir, H. Ding, S. Unarunotai, J. Rogers, M. Gillette, and G. Popescu, "Spatial light interference microscopy (SLIM)," Opt. Express 19, 1016-1026 (2011).

35. A. H. Maass and M. Buvoli, "Cardiomyocyte Preparation, Culture, and Gene Transfer," Methods Mol. Biol. 366, 321-330 (2007).

36. R. C. Gonzales and R. E. Woods, Digital Image Processing, 2nd ed., Prentice Hall, Englewood Cliffs, NJ (2002).

37. B. Alberts, Essential Cell Biology: An Introduction to the Molecular Biology of the Cell, Garland Publishers, New York (2004).

38. J. B. Pawley, Handbook of Biological Confocal Microscopy, Springer, New York (2006).

39. A. Einstein, "Investigations on the theory of the Brownian movement,"Ann. Physik. 17, 549-560 (1905).

40. M. T. Valentine, P. D. Kaplan, D. Thota, J. C. Crocker, T. Gisler, R. K. Prud'homme, M. Beck, and D. A. Weitz, "Investigating the microenvironments of inhomogeneous soft materials with multiple particle tracking," Phys. Rev. E 6406, 061506 (2001).
41. G. Popescu, T. Ikeda, R. R. Dasari, and M. S. Feld, "Diffraction phase microscopy for quantifying cell structure and dynamics," Opt. Lett. 31, 775-777 (2006).

42. V. Westphal, S. O. Rizzoli, M. A. Lauterbach, D. Kamin, R. Jahn, and S. W. Hell, "Video-rate far-field optical nanoscopy dissects synaptic vesicle movement,"Science 320, 246-249 (2008).

43. L. J. Millet, M. E. Stewart, J. V. Sweedler, R. G. Nuzzo, and M. U. Gillette, "Microfluidic devices for culturing primary mammalian neurons at low densities," Lab Chip 7, 987-994 (2007).

44. A. Y. N. Goldstein, X. N. Wang, and T. L. Schwarz, "Axonal transport and the delivery of pre-synaptic components,"Curr. Opin. Neurobiol. 18, 495-503 (2008).

45. C. F. Ibanez, "Message in a bottle: long-range retrograde signaling nervous system,"Trends Cell Biol. 17, 519-528 (2007).

46. J. R. Sotelo-Silveira, A. Calliari, A. Kun, E. Koenig, and J. R. Sotelo, "RNA trafficking in axons," Traffic 7, 508-515 (2006).

47. S. Sann, Z. P. Wang, H. Brown, and Y. S. Jin, "Roles of endosomal trafficking in neurite outgrowth and guidance,"Trends Cell Biol. 19, 317-324 (2009). 